\title{
The critical role of intracavity dynamics in high-power mode-locked fiber lasers
}

\author{
Brandon G. Bale ${ }^{a}$ and J. Nathan Kutz ${ }^{b}$ \\ ${ }^{a}$ Photonics Research Group, Aston University, Birmingham UK B4 7ET; \\ ${ }^{b}$ Department of Applied Mathematics, University of Washington, Seattle, WA 98195-2420
}

\begin{abstract}
We present a theoretical description of the generation of ultra-short, high-energy pulses in two laser cavities driven by periodic spectral filtering or dispersion management. Critical in driving the intra-cavity dynamics is the nontrivial phase profiles generated and their periodic modification from either spectral filtering or dispersion management. For laser cavities with a spectral filter, the theory gives a simple geometrical description of the intra-cavity dynamics and provides a simple and efficient method for optimizing the laser cavity performance. In the dispersion managed cavity, analysis shows the generated self-similar behavior to be governed by the porous media equation with a rapidly-varying, mean-zero diffusion coefficient whose solution is the well-known Barenblatt similarity solution with parabolic profile.
\end{abstract}

Keywords: Mode-locked lasers, high-power lasers, dispersion management

\section{INTRODUCTION}

Although the practical and innovative uses of mode-locked lasers has continued to grow in the past decade, ${ }^{1}$ its broader impact has been limited due to restrictions on pulse energies, which is a consequence of the underlying cavity nonlinearities. Recently however, great effort and progress has been made experimentally to achieve modelocked fiber lasers that produce high-energy, ultra-short pulses. ${ }^{2,3}$ A key parameter in achieving this aim is the cavity group velocity-dispersion (GVD). For anomalous GVD, ultra-short pulses can be easily obtained where the GVD balances the self-phase modulation (SPM) to produce soliton-like pulses that are nearly bandwidthlimited. ${ }^{1,4}$ The desire for higher energy pulses suggests consideration of cavities with segments of normal and anomalous GVD or with large and net normal GVD. These include the self-similar laser ${ }^{5}$ and the chirped pulse oscillator (CPO). ${ }^{6,7}$ In general, high-energy pulses can be generated, but it is necessary to compensate for the phase accumulated across the pulse. ${ }^{1,4}$

Recently, two new experimental configurations, both operating in the non-soliton regime, have been developed for obtaining high pulse energies. First, Chong et al. demonstrated a new class of high powered femtosecond fiber lasers, in which pulse-shaping is based on the spectral filtering of a highly-chirped pulse in the cavity. ${ }^{2,3}$ In contrast to soliton-like processes that dominate modern mode-locked lasers, these lasers depend strongly on dissipative processes as well as phase modulations to shape the pulse. Remarkably, no anomalous dispersion is required in the cavity, so this kind of laser is referred to as an all-normal dispersion (ANDi) laser. Second, self-similar (parabolic) pulse solutions have been recently observed experimentally ${ }^{8-10}$ in laser cavities with a mean-zero GVD. In both these new lasers, the intracavity dynamics, generated either from the spectral filtering or dispersion management, plays a critical role in both stabilizing the mode-locked pulse and producing highenergy output. The aim of this manuscript is to highlight the modeling efforts required for quantifying the large intracavity fluctuations and to show how these fluctuations are key to generating novel, high-energy, modelocked pulses. The analysis also provides a theoretical framework for optimizing the laser cavity performance and characterizing the global-attracting nature of the mode-locked solutions.

Further author information: (Send correspondence to J.N.K.)

J.N.K.: E-mail: kutz@amath.washington.edu, Telephone: 12066853029

Fiber Lasers VII: Technology, Systems, and Applications, edited by Kanishka Tankala, Jay W. Dawson, Proc. of SPIE Vol. $7580,75800 \mathrm{~W} \cdot($ C 2010 SPIE $\cdot$ CCC code: $0277-786 X / 10 / \$ 18 \cdot$ doi: $10.1117 / 12.840536$ 


\section{GOVERNING EQUATIONS}

The evolution of electromagnetic energy in the laser cavity is subject to a number of physical components: dispersion elements, bandwidth-limited gain components, and saturable absorption (intensity-discrimination) elements. These components are responsible for generating, among other things, intra-cavity chromatic dispersion, self-phase modulation, attenuation, and gain saturation. Haus proposed that these different elements could be averaged together into a single Ginzburg-Landau type evolution equation: the master mode-locking model. ${ }^{11}$ Due to stability considerations, the master equation is often augmented by a quintic saturation term which prevents blow-up of the solution. Thus the cubic-quintic Ginzburg-Landau equation (CQGLE) incorporates the laser cavity's intensity discrimination in a phenomenological way. The governing evolution is then given by

$$
i \frac{\partial u}{\partial z}+\frac{D}{2} \frac{\partial^{2} u}{\partial t^{2}}+(1+i \beta)|u|^{2} u+i \sigma|u|^{4} u+i \delta u-i g(z)\left(1+\tau \frac{\partial^{2}}{\partial t^{2}}\right) u=0,
$$

where the saturated gain behavior is given by ${ }^{1,4}$

$$
g(z)=\frac{2 g_{0}}{1+\|u\|^{2} / e_{0}} .
$$

Here $u$ is the electric field envelope, $z$ is the propagation distance, and $t$ is the retarded time. The energy of the pulse is given by $\|u\|=\int_{-\infty}^{\infty}|u|^{2} d t$, and $\beta$ and $\sigma$ measures the strength of the cubic and quintic saturable absorber terms respectively. The parameters $\delta, g_{0}$ and $e_{0}$ measure the cavity attenuation, the amplifier gain strength, and cavity saturation energy respectively.

\section{INTRACAVITY DYNAMICS}

The governing CQGLE (1) will be the starting point of the theoretical analysis of the intracavity dynamics. Specifically, we will consider two very different types of perturbations to the cavity: spectral filtering and dispersion management. Both compensate for accumulated phase and produce high-energy pulses whose dynamics can be elegantly captured with asymptotic and pertubation methods.

\subsection{Spectral Filtering}

The governing equation (1) is a partial differential equation modeling the spatial-temporal evolution of electromagnetic energy in the laser cavity. The variational method can be used to capture the intra-cavity pulse dynamics. The literature regarding variational reductions ${ }^{12}$ in nonlinear Schrödinger type systems is vast, especially given its applicability in optical transmission systems. To fully capture the varying phase profiles which have been observed in the ANDi laser cavity, ${ }^{2,3}$ we assume a solution-based mode-locking ansatz form

$$
u(z, t)=\frac{\eta(z)}{\sqrt{B(z)+\cosh (\eta(z) t)}} e^{-i \Psi(z, t)}
$$

with $\Psi(z, t)=A(z) \ln (B(z)+\cosh (\eta(z) t))+\varphi(z)$. The specific form of the phase profile $\Psi$ is essential to capture the different spectral profiles observed in the ANDi laser. ${ }^{2,3}$ The evolution of the ansatz parameters as a function of propagation distance ${ }^{12}$ is then found to satisfy the ordinary differential equations

$$
D \mathbf{x}=\mathbf{g}
$$

where $\mathbf{x}=\left[\eta_{z}, B_{z}, A_{z}, \varphi_{z}\right]^{T}, \mathbf{g}=\left[g_{1}, g_{2}, g_{3}, g_{4}\right]^{T}$, and

$$
D=\left[\begin{array}{cccc}
H & \eta H^{\prime} & 0 & 0 \\
(H-G) & -\eta\left(G^{\prime}+H^{\prime}\right) & 0 & 0 \\
0 & -A H^{\prime} & \frac{1}{2}(G-H) & -H \\
A H^{\prime} & 0 & \frac{1}{2} \eta\left(G^{\prime}+H^{\prime}\right) & -\eta H^{\prime}
\end{array}\right] .
$$




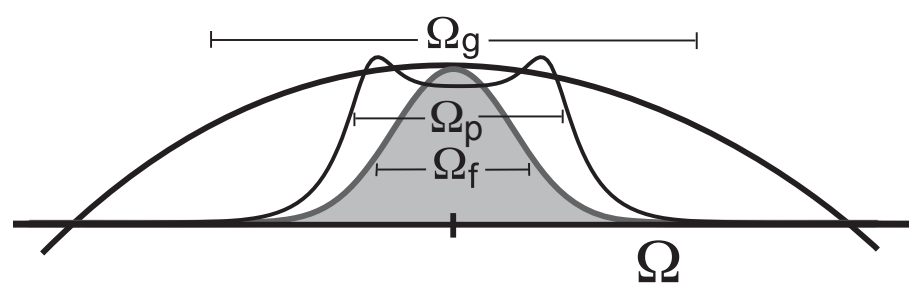

Figure 1. The experimental laser cavity configuration includes an amplifier with parabolic gain band-width $\Omega_{g}$ and a gaussian spectral filter with band-width $\Omega_{f}$. A typical pulse solution with spectral band-width $\Omega_{p}$ is also shown. Note that the key parameter $\Gamma=\Omega_{f} / \Omega_{p}$.

The components in the vector $\mathbf{g}$ include the terms from dispersion and self-phase modulation, as well as the gain and loss perturbations. They are given by

$$
\begin{aligned}
& g_{1}=2 \eta(g-\delta) H-2 \epsilon \eta^{3} H^{\prime}+\sigma \eta^{5} H^{\prime \prime}-\frac{1}{2} \tau g \eta^{3}\left(A^{2}+1\right) R \\
& g_{2}=\frac{D}{2} \eta^{3} A R-2 \eta(g-\delta) G-2 \epsilon \eta^{3} S-2 \sigma \eta^{5} Z+\frac{1}{2} \tau g \eta^{3}\left[3 W-2 Y-W A^{2}\right] \\
& g_{3}=\frac{3 D \eta^{2}}{4}\left(1+A^{2}\right) R+\frac{3 \eta^{2} H^{\prime}}{2}-2 A(g-\delta) H+\epsilon \eta^{2} A H^{\prime}-\frac{\sigma \eta^{4} A H^{\prime \prime}}{3}+\frac{\tau g \eta^{2} A}{2}\left(1+A^{2}\right) Q \\
& g_{4}=\frac{D}{4} \eta^{3}\left(1+A^{2}\right) R^{\prime}+\frac{\eta^{3} H^{\prime \prime}}{2}-2 \eta A(g-\delta) H^{\prime}-\epsilon \eta^{3} A H^{\prime \prime}+\frac{\sigma \eta^{5} A H^{\prime \prime \prime}}{3}-\frac{\tau g \eta^{3} A}{12}\left(1+A^{2}\right) R^{\prime},
\end{aligned}
$$

where all primes denote differentiation with respect to the parameter $B$, and the parameters $H, G, Q, R, S, W, Y$ and $Z$ are $B$-dependent integrals given by

$$
\begin{aligned}
& H=\int \frac{d t}{\Theta}, G=\int \frac{\ln \Theta d t}{\Theta}, Q=\int \frac{t \sinh ^{3} t d t}{\Theta^{4}}, R=\int \frac{\sinh ^{2} t d t}{\Theta^{2}} \\
& S=\int \frac{\ln \Theta d t}{\Theta^{2}}, W=\int \frac{\sinh ^{2} t \ln \Theta d t}{\Theta^{3}}, Y=\int \frac{\cosh t \ln \Theta d t}{\Theta^{2}}, Z=\int \frac{\ln \Theta d t}{\Theta^{3}}
\end{aligned}
$$

with $\Theta=B+\cosh t$ and all integrations range over $t \in[-\infty, \infty]$.

Despite the complicated structure of the equations, the dynamics are quite easy to characterize. The phase variable $\varphi$ can easily be eliminated from the system (4) resulting in a $3 \times 3$ system that can be analyzed in the phase plane. For different parameter regimes, the dynamics exhibits a stable node, a stable spiral node and a limit cycle in the $\eta, B$ and $A$ phase plane. The location of the fixed point as well as its stability depend on the parameters in the equations. The key contribution to pulse shaping in the ANDi lasers arises from the spectral filter. Although the fixed points of the reduced model have the correct temporal and spectral profiles seen in the ANDi laser, it fails to capture the round trip cavity dynamics. ${ }^{2,3}$ To capture the intra-cavity pulse fluctuations we must consider the operation of the spectral filter. The spectral filter can be assumed to be a Gaussian function with full width half maximum (FWHM) $\Omega_{f}$, and will typically fall under the gain bandwidth, as shown in Fig. 1. The ratio $\Gamma=\Omega_{f} / \Omega_{p}$, where $\Omega_{p}$ is the FWHM of the pulse bandwidth, determines how significant the filtering action is. For example, if $\Gamma>>1$, then the filter will have no effect on the pulse, however if $0<\Gamma<1$ then the filter will modify the pulse solution in some way.

To observe the spectral profile evolution per round trip in the laser cavity, the filter action cannot be averaged into Eq. (1) and must be considered as a discrete forcing on the governing equations. To obtain an understanding of the mechanism of the filter, we consider its effects in the context of the reduced model. For bandwidth ratios $0 \ll \Gamma<1$, multiplying the spectrum of (3) by a Gaussian filter results in a similar pulse form with modified 


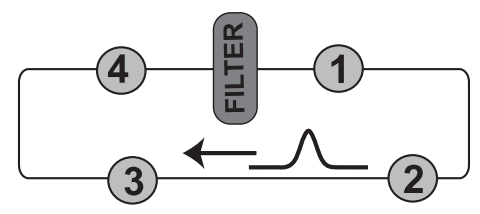

(a)

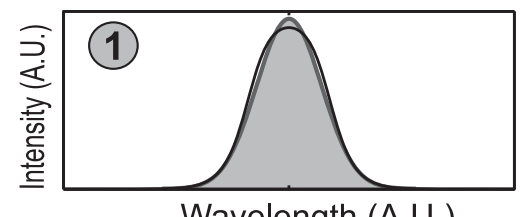

Wavelength (A.U.)

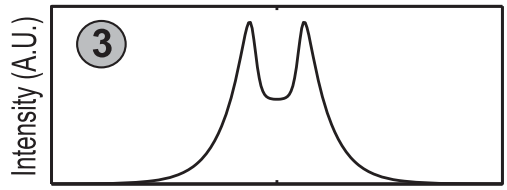

Wavelength (A.U.)
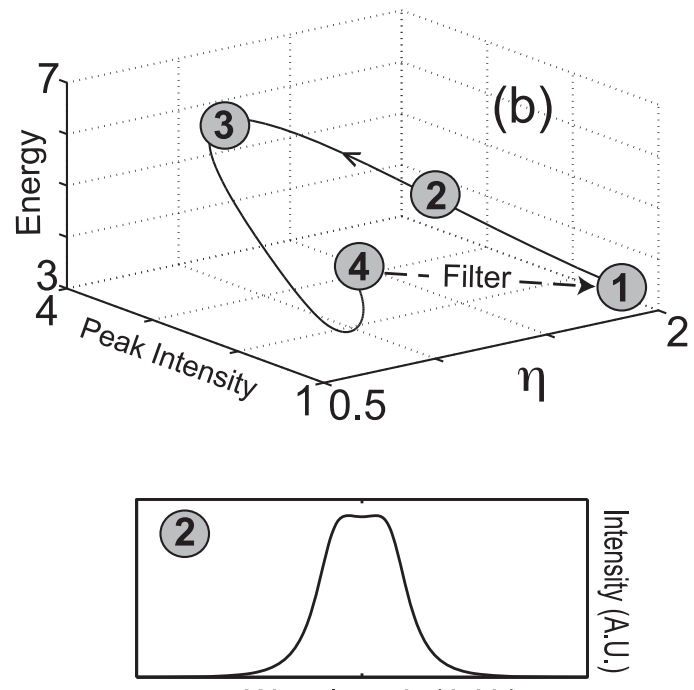

Wavelength (A.U.)

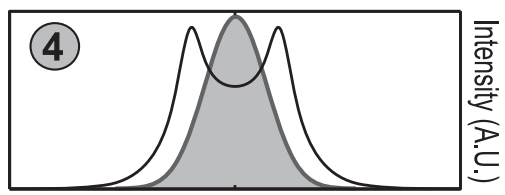

Wavelength (A.U.)

(c)

Figure 2. (a) Illustration of the laser cavity with discrete filter and four labeled positions in the cavity. (b) The intra-cavity mode-locked evolution in the experimentally relevant variables along with the action of the spectral filtering (dotted line). (c) The output spectral profiles at the labeled intra-cavity positions of (a) and (b). This prototypical pulse evolution is characteristic of the all-normal dispersion fiber laser. ${ }^{3}$ Note that high energy, high peak amplitude pulses can be obtained if the output coupler is placed at position 3 .

parameters. Thus we are able to assume that the filter acts only on the fixed point $\left(\eta_{0}, B_{0}, A_{0}\right)$ of the dynamical system (4), modifying the fixed point in some way, i.e. $\left(\eta_{0}, B_{0}, A_{0}\right) \rightarrow\left(\eta_{f}, B_{f}, A_{f}\right)$ where the new values are computed numerically after filtering via a least-square fitting. The accurate spectral and temporal fit between the post-filtered pulse and the pulse solution (3) with modified parameters clearly illustrates that the application of the spectral filter on the fixed point solution effectively changes the pulse parameters. Combining the reduced model, which is based on averaged evolution equations, with the essential discrete element in the laser, the spectral filter, we obtain a graphical interpretation of the intra-cavity dynamics of the ANDi laser since the filter acts as a periodic forcing (per round trip) on the governing equations that modifies the fixed point solution parameters $\left(\eta_{0}, B_{0}, A_{0}\right)$. We consider a laser configuration with the parameters $g_{0}=3, \delta=1, \tau=0.2, D=-0.4, \epsilon=0.5$, and $\sigma=-0.1$ corresponding to a stable node at $\left(\eta_{0}, B_{0}, A_{0}\right)=(1,-0.5,3.3)$.

By examining the evolution along flow lines we can understand the dynamics of the ANDi laser. Figure 2 shows an example of the laser dynamics with a filter bandwidth ratio $\Gamma=0.5$. Figure 2 (a) shows the laser configuration with the spectral filter as the primary discrete element. Figure 2(b) illustrates the phase line (whose initial condition is specified by the spectral filter width) in a relevant phase plane whose phase variables are the pulse duration $(\eta)$, peak amplitude $(\eta /(1+B))$, and pulse energy $\eta F(B)$. Figure $2(\mathrm{c})$ shows the spectral profiles at the various positions labeled in the laser set-up and phase plane. The fixed point is denoted by " 4 ", where the pulse directly after filtering is denoted by the " 1 " position. The periodic application of the filter (once per round trip) actively controls the parameters of the mode-locked pulse, changing the pulse solution parameters in (3) from the fixed point " 1 " to position " 4 ". Further, along the flow line the dynamic pulse evolution contains 
different spectral profiles that have been observed experimentally in the ANDi laser cavity. ${ }^{2,3}$ Note that the periodic orbit acts as a globally attracting state of the system, producing large intracavity fluctuations per round trip of the cavity. The optimal energy and peak power output can be extracted from the laser cavity at position " 3 " as is graphically demonstrated.

\subsection{Self-similar mode-locking}

In contrast to spectral filtering, we can also achieve the necessary phase compensation by applying a dispersion map to the laser cavity. This in turn will generate large intracavity fluctuations. In this case, the parameter $D$ in (1) is dependent upon $z$. We investigate (1) when the dispersion length $Z_{0}$ is much longer than the typical period $P$ of the dispersion map, so that

$$
\epsilon=P / Z_{0} \ll 1
$$

and the dispersion fluctuations occur on a rapid scale. The period $P$ is simply determined by the physical length of the laser cavity while the dispersion length is related to the pulse width of the mode-locked pulses. Specifically, the dispersion length is the length it takes for the full-width, half-maximum pulse width to double in the absence of nonlinearities. For convenience and simplicity, we let

$$
D=d(z / \epsilon)=\cos (2 \pi z / \epsilon) .
$$

Note that although the results apply to a general $d(z)$, it will prove helpful to consider the particular case here of a simple sinusoidal dispersion map.

Simulations suggest that the dispersion fluctuations must occur on a rapid-scale in order for the parabolic states to persist. Such a clear scale separation between the dispersion map period and the fundamental dispersion and nonlinearity scale suggests the application of a multi-scale transformation technique. The transformation procedure considered relies on the Green's function of the linear part of the left hand side of (1) since it accounts explicitly for the dispersion fluctuations. Using Fourier transforms, it is easy to calculate that the Green's function for the linear Schrödinger equation ${ }^{13}$

$$
i \mathbf{G}_{z}+\frac{1}{2} d(z / \epsilon) \mathbf{G}_{t t}=0,
$$

with $\mathbf{G}\left(t, t^{\prime}, 0\right)=\delta\left(t-t^{\prime}\right)$ is given by

$$
\mathbf{G}\left(t, t^{\prime}, z\right)=\frac{\exp (i \pi / 4)}{\sqrt{4 \pi \mu(z)}} \exp \left(\frac{-i\left(t-t^{\prime}\right)^{2}}{4 \mu(z)}\right) .
$$

Here $2 \mu(z)=\int_{0}^{z} d(s) d s \sim O(\epsilon)$ is the accumulated dispersion for a rapidly-varying, mean-zero map.

The transformation is performed by introducing the new function $A(t, z)$ defined by

$$
A(t, z)=\int \mathbf{G}^{\dagger}\left(t, t^{\prime}, z\right) u\left(t^{\prime}, z\right) d t^{\prime} .
$$

The evolution equation for $A$ can be found by using the adjoint relation $u(t, z)=\int \mathbf{G}(\xi, t, z) A(\xi, z) d \xi$. Plugging this into the governing equation (1), making use of (10), then multiplying by the adjoint $\mathbf{G}^{\dagger}(\xi, t, z)$ and integrating with respect to $\xi$ gives an exact transformation. At this point no approximations have been made - the transformation from $u$ to $A$ is simply a linear change of variables. Since $\mu \sim \epsilon \ll 1$, the integrals can be approximated using stationary-phase asymptotics. ${ }^{13}$ Expanding the integrals about the stationary phase points gives an approximate evolution for $A$ in terms as a series expansion in $\mu \sim \epsilon \ll 1$. Further, we assume that $\delta, \tau$, $\mu, \beta$, and $\sigma$ are small parameters thus allowing us to neglect higher order terms with $\mu \delta, \mu \beta$, etc., products.

The effective equation can be put into a more transparent form with the amplitude-phase decomposition $A(t, z)=\sqrt{\rho(t, z)} \exp (i \Theta(t, z))$ so that

$$
\begin{aligned}
& \rho_{z}=\mu(z)\left(\rho^{2}\right)_{t t}+2 \rho\left(\delta-\beta \rho-\sigma \rho^{2}\right)+\tau\left(\rho_{t t}-\frac{\rho_{t}^{2}}{2 \rho}-2 \rho \Theta_{t}^{2}\right) \\
& \Theta_{z}=-\rho-2 \mu(z) \rho \Theta_{t t}+\tau\left(\Theta_{t t}+\frac{1}{\rho} \rho_{t} \Theta_{t}\right) .
\end{aligned}
$$




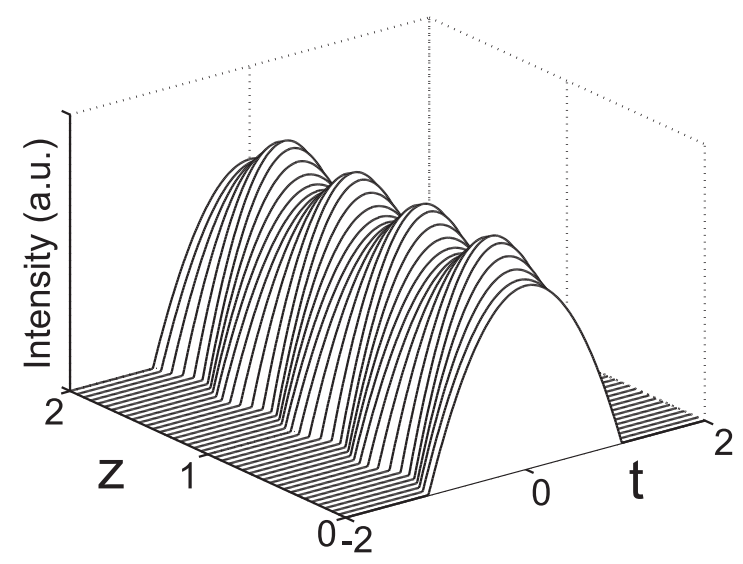

Figure 3. Typical evolution of the Barenblatt similarity solution (15) over four dipsersion map periods with $D(z)=$ $\cos (2 \pi z / \epsilon)$. The breathing dynamics is induced by the periodically varying diffusion coefficient $\mu(z) \sim O(\epsilon)$.

A key observation is that for $\mu>0$ the phase equation (13b) is ill-posed whereas for $\mu<0$ the amplitude equation (13a) is ill-posed. This problem is an artifact of the averaging process and can be treated via regularization or by including higher order correction terms. ${ }^{13}$ In contrast to other averaging techniques used on dispersion managed systems, we emphasize that the averaging technique used here retains the critical dependence of the parameter $\mu$ on $z$. This plays a key role in the stabilization of the parabolic state. Indeed, if the $\mu(z)$ parameter is averaged out to be a constant, the theory fails to correctly capture the breathing nature of the solutions. Specifically, the profile undergoes typical self-similar broadening until the expansion formally breaks down at $z \sim 1 / \sqrt{\epsilon}$. ${ }^{13}$

In the limit where the dissipative perturbations on the right hand side of (1) are small in comparison with the dispersion map, i.e. $(\delta, \beta, \sigma, \tau) \ll \epsilon<1$, the leading order amplitude equation is governed by the porous media equation

$$
\rho_{z}=\mu(z)\left(\rho^{2}\right)_{t t}
$$

The porous media equation has the Barenblatt similarity solution

$$
|u|^{2} \approx \rho(t, z) \sim \frac{1}{12\left(\gamma+z_{*}\right)^{1 / 3}}\left[a_{*}^{2}-\left(\frac{\left(t-t_{*}\right)}{\left(\gamma+z_{*}\right)^{1 / 3}}\right)^{2}\right]_{+}
$$

where $\gamma=\gamma(z)=2 \int_{0}^{z} \mu(s) d s$ and $f_{+}=\max (f, 0)$ so that the subscript + indicates that the function is either zero or positive in (15). The solution is characterized by the three parameters $\left(a_{*}, t_{*}, z_{*}\right)$ which represent the mass, center position, and pulse-width of the solution respectively. Note that $u \approx A$ when $\epsilon \ll 1$. ${ }^{13}$ Here, to first order in $\mu \sim \epsilon$, the evolution equation for the amplitude decouples from the equation for the phase. Figure 3 illustrates the typical time-dependent evolution of the Barenblatt solution (15) over four cavity round trips. We emphasize that the breathing dynamics results from the periodic fluctuations in the integral of the cumulative dispersion $\gamma(z)$. Indeed, the averaging technique used here retains the oscillatory nature of the dispersion map in the form of a $z$-dependent oscillatory coefficient in Eqs. (15). This oscillatory variation suppresses the structure from undergoing its usual self-similar broadening and allows for stable self-similar breathers.

Although the Barenblatt solution (15) captures the fundamental self-similar structure, it is not the attracting state of the underlying system. This is expected since we have neglected the dissipative terms needed to create an attractor. Further, the Barenblatt soluton has unphysical discontinuous derivatives at its edges. So although insightful, it is a mathematical idealization that is physically unrealizable. In many applications, spectral filtering is much weaker than other dissipative terms, i.e. $\tau \ll(\delta, \beta, \sigma, \mu)$. In this case, the amplitude equation

$$
\rho_{z}=\mu(z)\left(\rho^{2}\right)_{t t}+2 \rho\left(\delta-\beta \rho-\sigma \rho^{2}\right)
$$



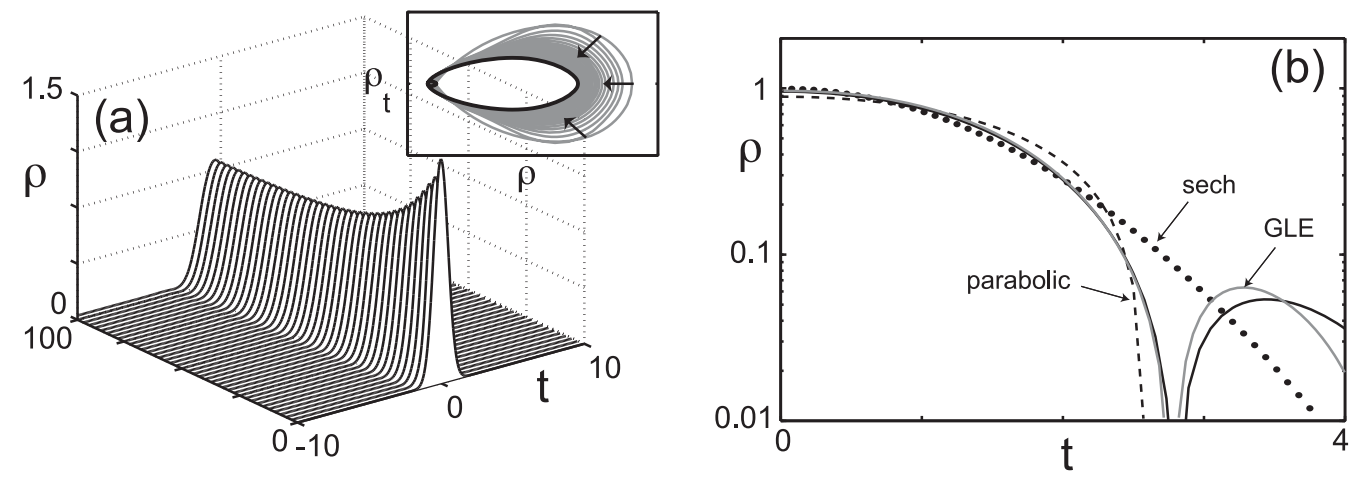

Figure 4. Attracting dynamics of the solution (a) and its phase-plane (inset) obtained from numerical simulation of the amplitude equation (16) from a Gaussian initial condition with $\delta=0, \beta=\sigma=0.1$, and $\epsilon=0.5$. The output is shown at the beginning of each dispersion map. (b) Comparison of the parabolic solution from solving (16) numerically (solid black) with the solution from the full governing Ginzburg-Landau equation (1) (solid grey), a quadratic Barenblatt profile (dashed) and hyperbolic secant pulse (dots). The tail structure is also exhibited in experiments. ${ }^{8}$

is still decoupled from the phase equation. Although exact solutions to (16) are not attainable, this equation sheds light on why parabolic states persist in this system. Specifically, for small values of the parameters $\delta, \beta$ and $\sigma$, equation (16) is perturbatively close to (14). Likewise, the solutions of the two equations should also be perturbatively close so that the leading order behavior of (16) inherits the self-similar Barenblatt structure of (15). Note that this implies that (16) is not strictly self-similar as certain symmetries associated with (14) are broken. Regardless, the inclusion of dissipative terms allows for an attracting parabolic breathers to exist for a wide range of parameter space. Further, numerical simulations suggest the parabolic states are robust to a variety of perturbations including white-noise fluctuations.

Figure 4 shows the numerical simulation of (16) from initial amplitude $\rho(t, 0)=\sqrt{2} \exp \left[-t^{2}\right]$. The output point in the Poincaré map is taken to be at the beginning of each map period. Figure 4(a) shows that the initial Gaussian structure quickly settles to a steady state solution in the Poincaré map. In contrast to the Barenblatt solution, the output pulse profile here has finite derivatives at its edges. The inset of Fig. 4(a) plots the corresponding $\left(\rho, \rho_{t}\right)$ phase plane and shows that there is indeed an attracting homoclinic orbit (solid line) which represents the steady state solution. To show that this attracting state has a parabolic profile, the output pulse (once settled to the parabolic breather), along with a Barenblatt quadratic (dashed) and hyperbolic secant (dotted) fit is plotted in Fig. 4(b). In addition, the numerical solution for the Ginzburg-Landau equation (1) with parameters $\tau=\delta=0,-\beta=\sigma=0.1$, and $\epsilon=0.5$ is included (solid grey). This shows that the solutions to (1) and (16) are perturbatively close as expected. Further, there is the remarkable agreement between the solution profile of (16) and experiments. ${ }^{8}$ Unlike the Barenblatt solution, the parabolic solution to (16) is a physically realizable, smooth profile that correctly captures the tail structure and attracting nature observed in experiments. ${ }^{8}$

\section{CONCLUSIONS}

In conclusion, we have shown that the consideration of the large intracavity dynamics generated from either spectral filtering or mean-zero, dispersion management leads to nontrivial, periodic mode-locked states that act as global attractors to the laser cavity system. In the all-normal dispersion fiber laser with filtering, we characterized these behaviors with a reduced model which is based on an averaged CQGLE equation. A key contribution to pulse shaping in these lasers arises from the spectral filter, which converts large frequency chirp to self-amplitude modulation. The variational method used here provides a geometrical interpretation that completely describes the intra-cavity dynamics. The resulting intra-cavity temporal and spectral profiles are in good agreement with observed numerical and experimental results. Thus the laser can be engineered to take advantage of the intra-cavity pulse dynamics by placing the output coupler at positions where the pulse has the 
desired temporal and spectral profile. When considering a rapidly-varying, mean-zero dispersion, the intracavity evolution dynamics results in a perturbed version of the nonlinear (porous media) diffusion equation with meanzero diffusion coefficient. The dissipative contributions in the GL equation make the parabolic structure an attracting state of the system. Thus the two driving mechanisms of parabolic propagation are the mean-zero dispersion map which generates self-similarity and dissipation which makes the self-similar structure an attractor. The combination of the two phenomena result in the formation of the parabolic breathers that have been recently observed experimentally in the context of mode-locked lasers. ${ }^{8}$

\section{acknowledgments}

The authors acknowledge insightful conversations about mode-locking performance with W. Renninger, A. Chong, and F. Wise. J. N. Kutz also acknowledges support from the National Science Foundation (DMS0604700) and the United States Air Force Office of Scientific Research (FA9550-09-0174).

\section{REFERENCES}

1. H. A. Haus, "Mode-Locking of Lasers," IEEE J. Sel. Top. Quant. Elec. 6, 1173-1185 (2000).

2. A. Chong, J. Buckley, W. Renninger, F. Wise, "All-normal-dispersion femtosecond fiber laser," Opt. Express 14, 10095 (2006).

3. A. Chong, W. Renninger, F. Wise, "Properties of normal-dispersion femtosecond fiber lasers," J. Opt. Soc. Am. B 25,140-148 (2008).

4. J.N. Kutz, "Mode-locked Soliton Lasers," SIAM Rev. 48, 629-678 (2006).

5. F. O. Ilday, F. W. Wise, and T. Sosnowski, "High-energy femtosecond stretched-pulse fiber laser with a nonlinear optical loop mirror," Opt. Lett. 27, 1531-1533 (2002).

6. A. Fernandez, T. Fuji, A. Poppe, A. Frbach, F. Krausz, and A. Apolonski, "Chirped-pulse oscillators: a route to high-power femtosecond pulses without external amplification," Opt. Lett. 29, 1366-1368 (2004).

7. V. L. Kalashnikov, E. Podivilov, A. Chernykh, and A. Apolonski, "Chirped-pulse oscillators: theory and experiment," Appl. Phys. B 83, 503-510 (2006).

8. F, Ö Ilday, J. R. Buckley, W. G. Clark, F. W. Wise, "Self-similar evolution of parabolic pulses in a laser," Phys. Rev. Lett. 92, 213902 (2004).

9. P. A. Belanger, "On the profile of pulses generated by fiber lasers:the highly-chirped positive dispersion regime (similariton)," Opt. Express 14, 12174-12182 (2006).

10. P. A. Belanger, "Stable operation of mode-locked fiber lasers: similariton regime," Opt. Express 15, 1103311041 (2007).

11. H. A. Haus, J. G. Fujimoto, and E. P. Ippen, "Structures for additive pulse mode locking," J. Opt. Soc. Am. B 8, 2068-2076 (1991).

12. D. Anderson, M. Lisak and A. Berntson, "A variational approach to nonlinear evolution equations in optics," Pramana J. Phys. 57, 917-936 (2001).

13. J. C. Bronski and J. N. Kutz, "Asymptotic behavior of the nonlinear Schrodinger equation with rapidlyvarying, mean-zero dispersion," Physica D 108, 315-329 (1997). 